\title{
An Improved Denoising Method Based on Stationary Wavelet Transform
}

\author{
Xiaoli Wang ${ }^{1,}$, , Yongfeng Dai ${ }^{2, b}$ \\ ${ }^{1}$ College of Mechanical \& Electronic Information, China University of Geosciences, Wuhan, Hubei \\ 430074, China; \\ ${ }^{2}$ School of power and energy engineering, Huazhong University of Science and Technology, \\ Wuhan, Hubei 430074, China. \\ acug_wxl@126.com, bdaiyf@163.com
}

\begin{abstract}
It is quite difficult to analyze experimental signals since they have low Signal-to-Noise Ratio (SNR). Discrete Stationary Wavelet Transform (SWT) can be used for signal denoising because of its energy concentration and shift invariance feature. this paper focuses on the noise reduction algorithms based on SWT and proposed a new threshold function for better denoising effect. The method is experimentally evaluated and simulated. The result shows that the proposed method is an effective tool for signal denoising.
\end{abstract}

Keywords: wavelet denoising, stationary wavelet transformation, threshold function, signal to noise ratio.

\section{Introduction}

All experimental signals come from various sensors of engineering environment. They are inevitably polluted by noise and interference. signals denoising technique is used to improve the reliability and accuracy of signal analysis. The core issue of signal denoising is to maximize signal to noise ratio (SNR).

Based on the discrete wavelet transform (DWT), various wavelet denoising methods like wavelet shrinkage, wavelet coefficient modeling, and wavelet transform modulus maxima denoising methods have been developed and shown to be more effective than traditional filtering methods $[1,2,3]$.

Donoho's hard and soft threshold [4,5] method is the most commonly used wavelet threshold denoising method. Although current wavelet-based denoising methods are chosen to increase SNR, inadequate noise removal and/or signal distortion comes with it.

The classical DWT suffers a drawback: DWT is not a time- invariant transform, which is a valuable property for signal analysis. This means that DWT brings out transform shift. The stationary wavelet transformation (SWT) [6] is proposed on the foundation of orthogonal wavelet transformation, which brings the properties of shift and scale invariance.

In this paper, an improved wavelet denoising approach is proposed based on SWT, that significantly improves denoising SNR and provides better implementation compared to previous DWT based methods. The study of the method concentrates on parts: SWT and the threshold function establishment.

The paper is organized as follows: First, a brief description of the wavelet-based denoising methods is provided. Second, the issues of SWT theory background and the improved threshold function are discussed. Third, experimental results are presented compared with classical wavelet. Finally, brief comments and conclusions are given.

\section{Theoretical Background}

\subsection{Stationary Wavelet Transform.}

Choose a mother wavelet $\psi(t)$ which satisfies the admissibility condition:

$$
C_{\psi}=2 \pi \int_{-\infty}^{\infty} \frac{|\psi(\omega)|^{2}}{\omega} d \omega
$$


Where $\psi(\omega)$ is the Fourier transform of $\psi(t)$.

The wavelet transforms of a signal $x(t)$ is defined by:

$$
W(b, a)=\int_{-\infty}^{\infty} x(t) \frac{1}{\sqrt{a}} \psi^{*}\left(\frac{t-b}{a}\right) d t
$$

Where $a$ is the scale parameter, $b$ is the shift parameter, $\psi^{*}(t)$ is the complex conjugate of mother wavelet $\psi(t)$.

Take the discrete value of the scale and the shift parameter respectively: $a=2^{j}, \quad b=2^{j} k$. The discrete wavelet transforms (DWT) of $x(t)$ can be defined as:

$$
W(k, j)=\int_{-\infty}^{\infty} x(t) \frac{1}{\sqrt{2^{j}}} \psi^{*}\left(2^{-j} t-k\right) d t
$$

Mallat algorithm of DWT is complemented by combining high-pass filters $H_{j}$ and low-pass filters $L_{j}[3]$ shown in fig. 1 .

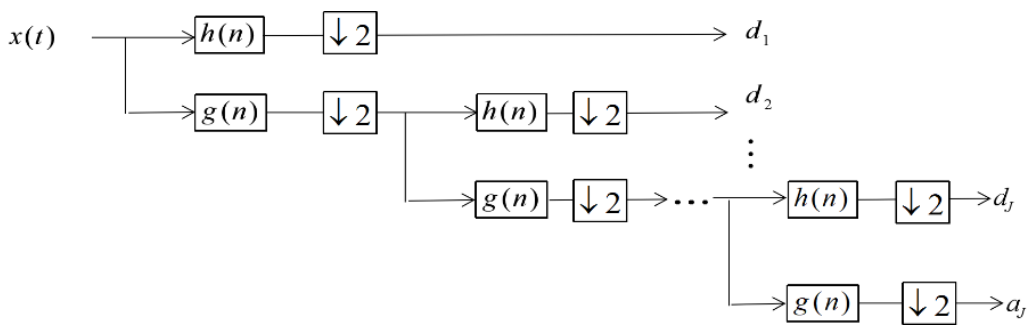

Fig.1 Mallat's algorithm of DWT

However, down-Sampling is carried out in Mallat's algorithm complementation. It makes the classical DWT not a time-invariant transform. the SWT makes it possible to perform time-invariant transform by up-sampling the filter coefficients of the high-pass filters and the low-pass filters.

In the SWT [6], as shown in Fig. 2, the coefficients of the high-pass filters and the low-pass filters are up-sampled, The wavelet coefficients are given by the sequences $d_{j}$ and the scaling coefficient is given by the sequence $a_{j}$. Where $\mathrm{j}$ represents the order of SWT.

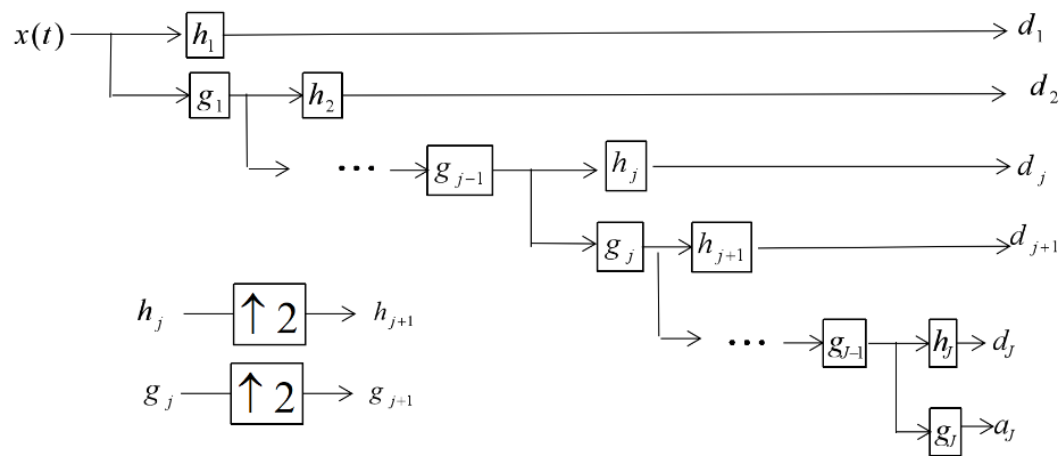

Fig.2 Stationary wavelet transform

Suppose $g_{1}(n)=<\phi, \phi_{-1, n}>, \quad h_{1}(n)=<\psi, \phi_{-1, n}>, \quad \phi$ is the scale function; $\psi$ is the wavelet function. The decomposition expression of stationary wavelet transformation is:

$$
a_{j, k}=\sum_{n} g_{j}^{\uparrow}(n-2 k) a_{j-1, n}, \quad d_{j, k}=\sum_{n} h_{j}^{\uparrow}(n-2 k) d_{j-1, n}
$$

Where, $a_{j, k}$ denotes scale coefficient of approach part, $d_{j, k}$ denotes wavelet coefficient of detail part. $g_{j}^{\uparrow}$ and $h_{j}^{\uparrow}$ denotes the interpolation of $g_{j-1}$ and $h_{j-1}, \mathrm{n}=0,1, \ldots, \mathrm{N}-1, \mathrm{~N}$ is the signal length.

Reconstruction expression of wavelet transformation is: 


$$
a_{j-1, n}=\frac{1}{2} \sum_{k}\left[g_{j}^{\prime}(n-2 k)+g_{j}^{\prime}(n-2 k-1)\right] a_{j, k}+\frac{1}{2} \sum_{k}\left[h_{j}^{\prime}(n-2 k)+h_{j}^{\prime}(n-2 k-1)\right] d_{j, k}
$$

Where $g^{\prime}$ and $h^{\prime}$ are the dual base of $g$ and $h$ respectively.

After applying to wavelet transform, the wavelet coefficients of the signal and noises represent different characteristics in the wavelet domain. the signal is coherent and owns energy concentration property, which gathers the signal in just a few high magnitude coefficients, whereas noise is incoherent and represented by a large number of coefficients with small magnitudes. Denoising is complemented by setting noise coefficients to zero or comparatively small, while keeping the signal coefficients.

\subsection{Proposed Threshold Function.}

Donoho's hard and soft threshold [4,5] function is the most commonly used approach, they have their own characteristics and limitations. Wavelet coefficients processed by hard threshold function hurts the signal continuity, which brings out the oscillation and poor smoothness of the reconstructed signal. Meanwhile, soft threshold function brings out the distortion of the reconstructed signal [7].

The improved threshold function is set as follows:

$$
d_{i}=\left\{\begin{array}{rr}
d_{i}-\alpha \frac{\lambda_{i}^{4}}{d_{i}^{3}} & \left|d_{i}\right| \geq \lambda_{i} \\
(1-\alpha) \frac{d_{i}^{5}}{\lambda_{i}^{4}} & \left|d_{i}\right|<\lambda_{i}
\end{array}\right.
$$

Where $\alpha$ is between 0 and 1 .

The proposed threshold function is shown in Fig.3, compared with soft and hard threshold function, the coefficients below the threshold are tuned by a power function instead of simply setting to zero. Furthermore, it is also continuous to weaken the oscillation and poor smoothness of the reconstructed signal.

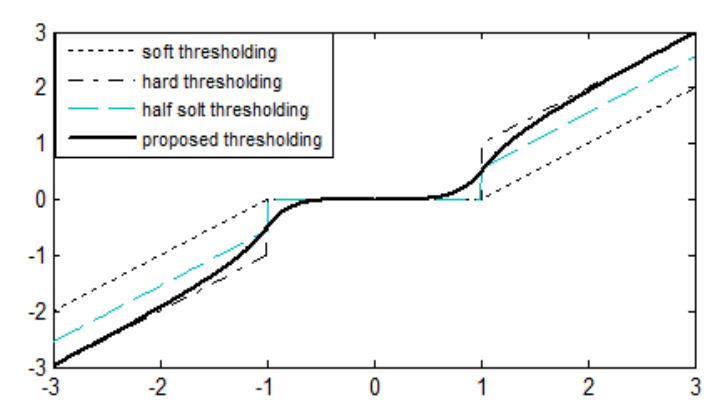

Fig. 3 comparison with hard and soft threshold function

\subsection{Steps of Denoising Method}

The SWT based method provides effective signal denoising with minimum computational complexity. The steps of the method are as follows:

Input the signal

Use the Least Square Method to remove the trend noise.

Select a wavelet and determine the decomposition level $\mathrm{J}$ for denoising.

Take the $J$ level discrete stationary wavelet transform (SWT) of the signal, referred to as detail components of each level and the $\mathrm{J}^{\text {th }}$ approximation component.

Estimate the noise variance and calculate the noise threshold for the each detail components.

Apply proposed threshold function to the detail components.

Take the inverse discrete wavelet transform (ISWT) . 


\section{Experimental Result}

The wavelet denoising evaluation criterion includes two indexes: Signal Noise Ratio (SNR) and Root Mean Squared Error (RMSE), the equations are as:

$$
S N R=10 \lg \left(\text { power }_{\text {sig }} / \text { power }_{\text {noise }}\right), R M S E=\sqrt{\frac{1}{N} \sum_{n=1}^{N}[x(n)-\hat{x}(n)]^{2}}
$$

Where $\mathrm{N}$ is the signal length, power $_{\text {sig }}$ is the desired signal power, power $_{\text {noise }}$ is the noise power, $x(n)$ is the original signal, $\hat{x}(n)$ is the polluted signal.

According to the definitions of SNR and RMSE, the larger the SNR, the smaller the RMSE and the better the noise reduction effect .

To prove the effectiveness of the proposed method. the tested signal is constructed, which contains three parts: sinusoidal signal, trend noise as well as random noise. the signal-to-noise ratio is $5 \mathrm{db}$, use the SWT and the improved threshold function. threshold selection is based on the stein's unbiased risk estimate, takes decomposition layer $\mathrm{J}=3$. The proposed threshold function in this paper is adopted. The result is shown in fig.4.
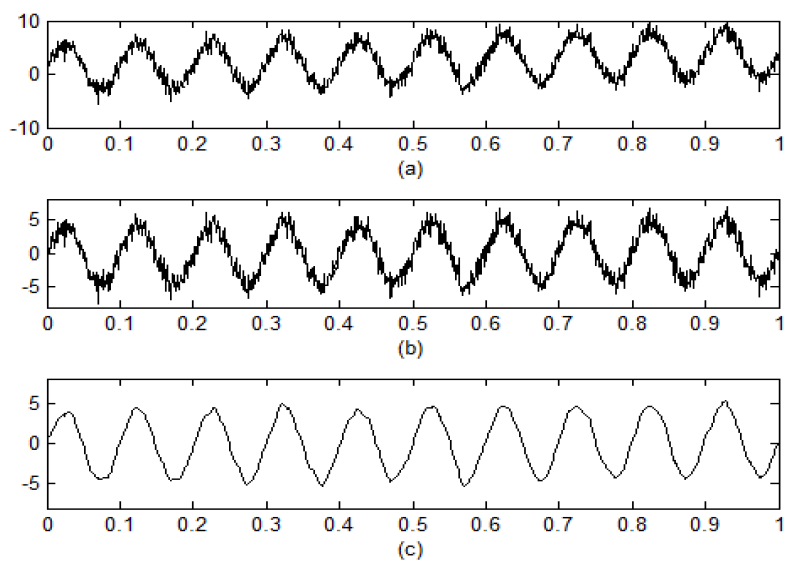

(a)the polluted signal; (b)signal after Least Square Method;(c) signal after SWT denoising

Fig. 4 reveals that the signal restores the primary signal approximately, the signal smoothness is greatly improved after application of the SWT as well as the proposed threshold function.

To further prove the effectiveness of the proposed method. SNR and RMSE are calculated to the polluted signal with SNR varies from $5 \mathrm{~dB}$ to $30 \mathrm{~dB}$. The comparison is also made between the SWT and DWT. The result is shown is fig.5.
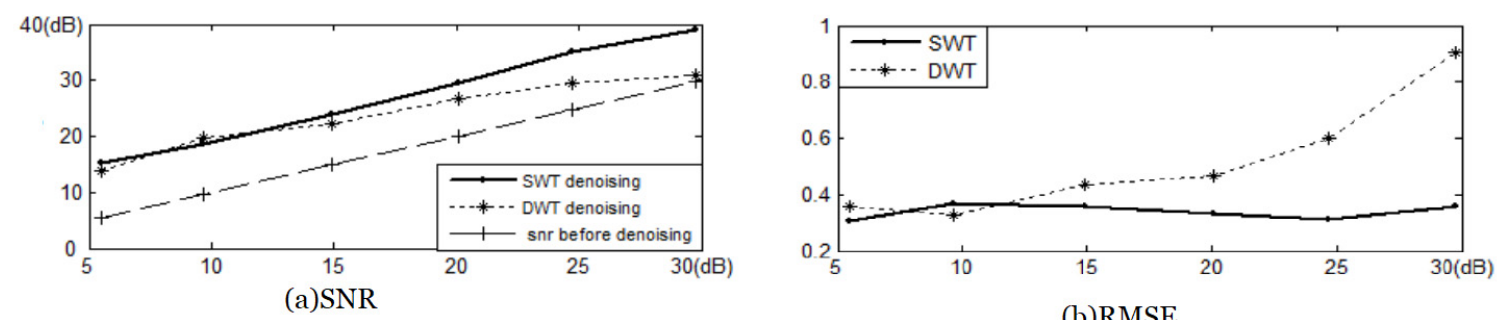

(b)RMSE

Fig.5 (a)SNR comparison between SWT and DWT denoising

(b)RMSE comparison between SWT and DWT denoising

The result indicates that the proposed SWT based denoising method accomplishes higher SNR and lower RMSE most of the case. 


\section{Summary}

Stationary wavelet transformation has excellent benefits of revolving, translation and scale invariable. the paper applies SWT in signal denoising and proposed a new threshold function. The experimental result demonstrates that signal denoising algorithm based on SWT is effective and feasible.

\section{References}

[1]. T. Cai, B. W. Silverman. Incorporating information on neighbouring coefficients into wavelet estimation. Sankhya, Indian J. Statist. B, vol.63(2001), No. 2, p. 127-148.

[2]. S. G. Chang, B. Yu, and M. Vetterli. Spatially adaptive wavelet thresholding with context modeling for image denoising. IEEE Trans. Image Process. vol. 9(2000), No. 9, p. 1522-1531.

[3]. S. G. Mallat. A theory for multiresolution signal decomposition: The wavelet representation. IEEE Trans. Pattern Anal. Mach. Intell. vol. 11(1989), No. 7, p. 674-693.

[4]. Donoho D L. Denoising by soft threshold. IEEE Transaction On Information Theory.1995,41(3), p.613-627.

[5]. Donoho, D.L. I.M. Johnstone. Ideal spatial adaptation by wavelet shrinkage. Biometrika, vol. 81(1994), p. 425-455.

[6]. Nason G.P, Silverman B.W. The stationary wavelet transforms and some statistical applications. Lect. Notes Stat., 1995, p.103.

[7]. J. Li, C. Cheng, T. Jiang, S. Grzybowski. Wavelet de-noising of partial discharge signals based on genetic adaptive threshold estimation. IEEE Transactions on Dielectrics and Electrical Insulation, vol.19(2012), No. 2, p.543-549. 